El método de investigación acción en el área educativa:

Un ejercicio pertinente e inclusivo para los formadores del siglo XXI.

Monica Chicaiza Rey - Yesenia Martelo - Ronal Blanquicett Narváez (84:94)

\title{
EL MÉTODO DE INVESTIGACIÓN ACCIÓN EN EL ÁREA EDUCATIVA: UN EJERCICIO PERTINENTE E INCLUSIVO PARA LOS FORMADORES DEL SIGLO XXI
}

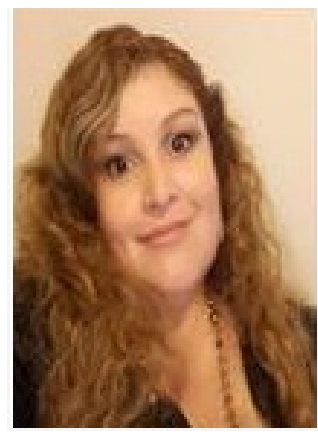

\author{
Monica Chicaiza Rey \\ I.E.M INEM Pasto, Colombia \\ Monitarey433@gmail.com
}

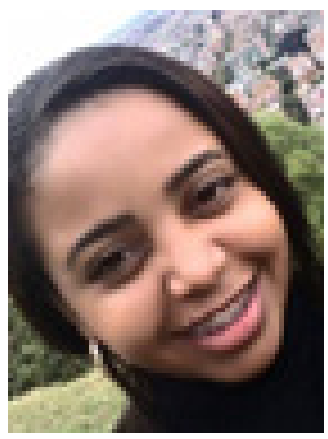

\section{Yesenia Martelo}

Institución Educativa Palmira, Córdoba, Colombia

yesenia722@gmail.com

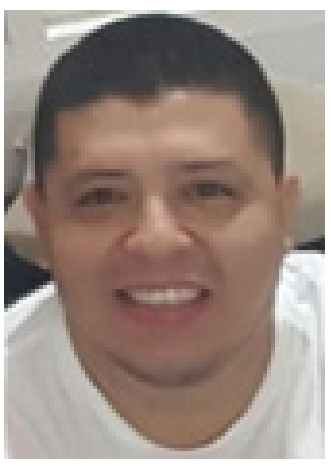

\section{Ronal Blanquicett Narváez \\ Institución Educativa La Ribera, Córdoba, Colombia \\ ronalabn@gmail.com}

\section{RESUMEN}

El presente trabajo es un análisis del método de investigación de acción en relación con sus contribuciones al área de la educación. Comienza con la problematización de la práctica educativa realizada por los docentes, como principio fundamental para desarrollar un proceso educativo en contexto; es decir, coherente e inclusivo. Del mismo modo, la disertación finaliza describiendo las ventajas que tiene la investigación acción para mejorar la práctica educativa, así como la pertinencia de este método para la formación de niños y jóvenes con las habilidades necesarias 
para desempeñarse en la sociedad del siglo XXI, que está en constante cambio.

Palabras clave: investigación acción, educación, formación, pertinencia, inclusión.

\title{
THE ACTION RESEARCH METHOD IN THE EDUCATIONAL AREA: A PERTINENT AND INCLUSIVE EXERCISE FOR 21ST CENTURY TRAINERS
}

\begin{abstract}
The current paper, is an analysis of the action research method in relation to its contributions to the area of education. It starts with the problematization of the educational practice done by teachers, as a fundamental principle to develop an educational process in context; that is, coherent and inclusive. In the same way, the dissertation ends by describing the advantages action research has, to improve the educational practice. Thus, the relevance of this method when training children and young people with the necessary skills to perform in the 21 st century society, which is in constant change.
\end{abstract}

Key Words: action research, Education, training, relevance, inclusión.

\section{INTRODUCCIÓN}

El presente ensayo tiene como propósito realizar un acercamiento al método investigación acción y su aplicación en la investigación en el área educativa, tomando como punto de partida una breve descripción de la relevancia de los procesos investigativos para la educación, así como las bases epistemológicas que sustentan este tipo de método de investigación.

Posteriormente se encaminará la reflexión hacia algunos tópicos relacionados con los aportes de la investigación acción en el ámbito de la investigación educativa, los problemas que resuelve, las ventajas que le confiere al docente investigador, así como las habilidades que requiere y la pertinencia de este método en la sociedad de cambio constante del siglo XXI.

\section{DESARROLLO}

El desarrollo de la profesión educativa en la sociedad de la información es un reto y un compromiso que demanda calidad, coherencia y mejoramiento continuo para poder responder a las necesidades de formación del contexto y estar a la vanguardia de los vertiginosos cambios que se presentan en un mundo globalizado, para ello es necesario que constantemente la pedagogía aspire incrementar su lucidez (Furlán et al., 1993) a través de la puesta en marcha de un ejercicio 
El método de investigación acción en el área educativa:

Un ejercicio pertinente e inclusivo para los formadores del siglo XXI.

Monica Chicaiza Rey - Yesenia Martelo - Ronal Blanquicett Narváez (84:94)

investigativo cuyo objetivo radique en "indagar acerca de la verdad de sus teorizaciones, de los conceptos que soportan sus postulados, de lo que enarbola para su justificación como disciplina académica" (ibíd.) en la sociedad que le confiere el transcendental encargo social de formar a sus ciudadanos, es así como en este escenario los procesos investigativos son de vital importancia si se pretende avanzar desde el marco de la pertinencia de lo contrario la práctica educativa se convertirá en un accionar anacrónico y poco significativo.

Forlán y Pasillas (1993) en su artículo investigación, teoría e intervención en el campo pedagógico tipifica dos grandes grupos de investigaciones en el ámbito educativo, la primera investigación pedagógica y la segunda el estudio de las prácticas educativas cuyo propósito consiste en "elucidar el carácter social, psicológico, antropológico, político, lingüístico, histórico de las acciones de influencia socialmente legitimadas como educación" (ibíd.) siendo este último grupo el objeto de análisis de la presente disertación por ser la práctica educativa el objeto de estudio de la investigación acción Colmenares y Piñeros (2008)

Así mismo, las investigaciones de las prácticas educativas se enmarcan en el enfoque epistemológico denominado por Padrón (2007) empirista idealista también categorizado como enfoque introspectivo-vivencial (Sirit \& Enrique, 2014) en el que se indica que "la vía más apropiada para acceder al conocimiento es una simbiosis entre el investigador y su objeto de estudio; donde el objeto pasa a ser una experiencia vivida, sentida y compartida con el investigador" que en palabras de Quine (1969) citado en Padrón (2007) se resumirá en la siguiente premisa: no hay conocimiento previo a la experiencia.

De manera que, esta plataforma epistemológica las investigaciones en educación pueden alinearse a tendencias cualitativas entre las cuales surge el método investigación - acción como un nuevo camino para la investigación del profesorado. Es por ello que cuando se desarrollan estudios investigativos en el área de la educación, éste tipo de enfoques metodológicos poseen especial importancia, puesto que, permite al docente investigador observar la realidad investigada y los comportamientos directos, de quien es investigado, pudiendo con ello tomar la información de la fuente como se origina y a partir de allí, sistematizar, analizar y emitir juicios, recomendaciones y conclusiones sobre un objeto de investigación.

Se puede aseverar que la investigación acción es un método de investigación significativo para la comunidad educativa por cuanto su aplicación permite desarrollar y profundizar la comprensión de la problemática en lo que se refiere a su rol docente, Latorre, A.(2005) explica que la investigación acción se utiliza para describir una familia de actividades que realiza el profesorado 
en sus aulas con fines, tales como: el desarrollo curricular, su autodesarrollo profesional, la mejora de los programas educativos, los sistemas de planificación o la política de desarrollo. De la misma forma, la investigación-acción pretende una mejor comprensión, sistematización, análisis y estudio de la realidad social. En este sentido, algunas de las principales aplicaciones de este tipo de investigación son, en palabras de Pérez Serrano (1990): Desarrollar estrategias y métodos para actuar de un modo más adecuado, descubrir espacios donde se pueda fomentar el desarrollo social de la comunidad, facilitar dinámicas de trabajo adecuadas para la constitución de grupos sociales, proporcionar técnicas e instrumentos de análisis de la realidad, procedimientos de recogida y análisis de los datos e iluminar todo el accionar desde la óptica de la investigación cualitativa, al vincular en el proceso, a un mismo tiempo, la investigación y la acción, la teoría y la praxis.

En consecuencia, los aportes de la investigación - acción son numerosos y significativos y cuentan con un amplio abordaje en variados estudios de la literatura actual, siendo conveniente para alcanzar el objetivo del presente análisis solo considerar algunas aplicaciones específicas del método investigación - acción en la investigación educativa por lo que a partir de esta sección se tendrán como variables los tópicos relacionados con la problematización de la práctica educativa, la coherencia e inclusión de la investigación educativa.

Por tanto, a problematización de la práctica educativa es quizás la aplicación que más aporta este método a los procesos investigativos en educación debido a que "la investigación -acción contempla los problemas desde el punto de vista de quienes están implicados en ellos"(John Elliott, 2005), es decir, que a partir de esta variable se establece que el insumo para el ejercicio investigativo es la vivencia misma del docente convirtiendo el quehacer diario del profesorado en un punto de partida y antesala de transformación de nuevos conocimientos, para Latorre (2001), "el profesorado como investigador formula nuevas cuestiones y problematiza sus prácticas educativas" posicionándose como gestor de la tarea investigativa y "evitando así la elección errónea de problemas de investigación, debido a que quienes trabajan en la teoría están tratando de responder a preguntas que en realidad no se han hecho los que trabajan en la práctica” (ibíd.) por ello el ejercicio de problematización que implica este método es fundamental para realizar una investigación pertinente y asertiva que apunte a resolver los problemas reales que se presentan en los escenarios educativos.

Esta primera etapa de problematización de la práctica educativa es propuesta por Lewis precursor de la investigación - acción quien a su vez propone un ciclo espiral de acciones que equipara con el método científico (John Elliott, 2005) donde el diagnóstico o reconocimiento de una situación problemática en la práctica docente correspondería a la observación con la notoria 
El método de investigación acción en el área educativa:

Un ejercicio pertinente e inclusivo para los formadores del siglo XXI.

Monica Chicaiza Rey - Yesenia Martelo - Ronal Blanquicett Narváez (84:94)

diferencia que en la investigación - acción, la fase inicial se deriva de la autorreflexión del maestro el cual hace parte de la realidad educativa que se quiere investigar, es decir, el objeto de estudio la investigación acción es "explorar los actos educativos tal y como ocurren en los escenarios naturales dentro y fuera del aula" (Colmenares y Piñeros 2008).

Desde esta mirada problemátizadora Colmenares y Piñero (2008) consideran la investigación - acción como una herramienta epistémica orientada al cambio educativo:

"Por cuanto, se asume una postura ontoepistémica del paradigma socio-crítico, que parte del enfoque dialéctico, dinámico, interactivo, complejo de una realidad que no está dada, sino que está en permanente deconstrucción, construcción y reconstrucción por los actores sociales, en donde el docente investigador es sujeto activo en y de su propia práctica indagadora”

$\mathrm{Y}$ es en ese ser y estar del docente donde "las cuestiones de investigación surgen de la experiencia cotidiana, de las discrepancias entre lo que se pretende y lo que ocurre en clase" (Latorre 2001) sin embargo el ejercicio problemátizador no excluye perse a la teoría aunque el investigador en la acción comienza con un problema práctico este también es de tipo teórico, la teoría con frecuencia está incluida en su propia práctica y no ha sido formulada de manera consciente (John Elliott, 2005) en este sentido se construye un conocimiento educativo, validado en la práctica (Latorre 2001) donde se pasa de un conocimiento práctico más bien inconsciente a un conocimiento crítico y teórico construido a través del dialogo y la interacción con colegas y estudiantes (Schon, 1987 citado en Colmenares y Piñeros 2008).

De manera que, la investigación acción en la investigación educativa en párrafos anteriores implícitamente se denota que evita el desenfoque al establecer como punto de partida la problematización de la praxis docente. No obstante, este método va más allá de la identificación de situaciones problema porque también soluciona algunas dificultades de la metodología cualitativa relacionados con la apatía y desinterés de las personas que hacen parte del grupo investigador debido a que este método permite a los miembros de la comunidad investigativa aprender como conducir investigación y valorar el papel que la investigación puede jugar en sus vidas (Balcázar, 2003) generando procesos de empoderamiento en la búsqueda de construcción de conocimiento educativo que desencadenan mayor cohesión social al "desarrollar un mayor sentido de pertenencia lo que aumenta su grado de compromiso y control de la investigación” (ibíd.) en los grupos investigativos otorgando un mayor significado a los resultados obtenidos debido a que son fruto del esfuerzo colectivo, en un sentido más romántico y en palabras del Balcázar (2003) La IA tiene fe en las personas y en su capacidad para participar en el proceso de investigación. 
En suma, otro problema que resuelve la investigación - acción es el de darle sustento epistemológico al investigador educativo quitando todo temor de que el conocimiento construido no tenga validez ya que al derivar de la metodología cualitativa la cual es una "opción metodológica válida para el abordaje de los problemas socioeducativos"(Colmenares y Piñeros 2008) se desarrolla sobre las bases de un "proceso activo, sistemático y riguroso de indagación dirigida, en la que se toman decisiones sobre lo investigable en tanto se está en el campo de estudio" (Pérez Serrano 1994 citado en Colmenares y Piñero 2008) es así como el conocimiento educativo resultado de la construcción realizada en el marco de la investigación -acción puede considerarse "científico" en la medida que en concordancia con Padrón (2014) cumpla con las propiedades de sistematización, socialización y fundamentación teórica que se plantean como criterio de demarcación para la validación de lo científico, ahora, para ahondar un poco más y resaltar el carácter inclusivo que caracteriza esta metodología es pertinente traer a colación el siguiente fragmento de las notas sobre enfoques epistemológicos, estilos de pensamiento y paradigmas de José Padrón:

"De ese modo, mediante este triple criterio de sistematización, socialización y fundamentación teórica, podremos liberarnos tanto de la relatividad de los controles de poder (los cuales nos obligan a considerar como 'ciencia' sólo aquello que ellos nos dicen que es) como de las limitaciones de los criterios lógico-metodológicos (que, al fin y al cabo también son relativos a una determinada socio-cultura). Es por eso por lo que, con estos criterios, podemos hablar justificadamente de la 'Ciencia Precolombina' (azteca, inca, etc.) o de la 'Ciencia del Campesino', aunque no sea la misma que la actual cultura occidental considera como tal." (Padrón, 2014)

Bajo esta fundamentación y en este orden de ideas la investigación - acción rompe el mito que el accionar investigativo solo puede ser desarrollado en altas esferas académicas y en laboratorios de alta tecnología solo por grandes eruditos de las diferentes ciencias y en este punto se señala lo inclusivo como una característica inherente de este método de investigación.

Como es evidente, a las ventajas que confiere al docente investigador la investigación acción se hace inevitable desde la concepción integradora de la enseñanza como actividad investigadora (Latorre, 2001) extender este análisis hacia los beneficios de este método para la práctica educativa y la profesionalización del docente.

Al sumergirse en los procesos de la investigación - acción el docente adquiere mayor autonomía y se libera de las cadenas del enfoque de la racionalidad técnica donde es considerado un simple consumidor de teorías así desde la plataforma emancipadora de este método el profesor investigador "asume más control sobre su vida profesional y desarrolla su juicio profesional 
El método de investigación acción en el área educativa:

Un ejercicio pertinente e inclusivo para los formadores del siglo XXI.

Monica Chicaiza Rey - Yesenia Martelo - Ronal Blanquicett Narváez (84:94)

logrando autonomía y emancipación" (Latorre 2001). Superando los obstáculos burocráticos que menoscaban y erosionan su magna profesión.

Como consecuencia de la autonomía docente se resignifica el papel de el mismo en el sistema educativo convirtiéndose en un sujeto activo gestor de transformaciones y a su vez en un "aprendiz de largo alcance, como aprendiz de por vida" por preocuparse por transformar permanente y sistemáticamente su práctica pedagógica. (Colmenares y Piñero 2008) en palabras de Latorre (2001) el maestro investigador que se profesionaliza a medida que investiga su práctica y este nuevo rol del profesorado propicia mejores condiciones para la práctica educativa y las instituciones educativas traduciéndose en calidad la cual solo es posible cuando se apoya en los resultados de la investigación y a su vez esta encuentra en la practica un canal y espacio natural para indagar, analizar y aplicar sus resultados. (Latorre 2001).

En esta perspectiva del nuevo rol docente - investigador y desde una dimensión emancipadora del método reflexión - acción se hace necesario que el profesorado desarrolle habilidades relacionadas con el pensamiento crítico que le permita cuestionar su quehacer diario, así como la relación y/o coherencia de las teorías que le imponen con el desarrollo de su práctica educativa.

Sin embargo, el desarrollo del pensamiento crítico va más allá de la práctica in situ en la escuela superando en el análisis de las situaciones las barreras institucionales siendo la investigación acción un método que "genera conciencia política entre los participantes en el proceso"(Balcázar, 2003) y representa creencias sobre el papel del científico social para disminuir la injusticia en la sociedad. (ibíd.) es así como alejada de toda racionalidad técnica este tipo de metodologías fortalecen el papel del docente como profesional autónomo.

Finalmente, exponiendo algunos argumentos de como la puesta en marcha de investigación -acción en el ámbito educativo le otorga el estatus de pertinente a la práctica educativa. En primer lugar, explayaremos la conceptualización de lo que concierne pertinente que en su momento describió Teodoro Pérez (2009) siendo gerente del plan decenal de educación 2006-2016 del Ministerio de Educación de Colombia:

"la educación es pertinente cuando guarda congruencia (Es decir, conveniencia, coherencia y relación lógica) con las condiciones y necesidades sociales, con las normas que regulan la convivencia social y con las características concretas de los educandos en sus diversos entornos naturales y sociales de interacción ”(Pérez, 2009.) 
Efectivamente gracias a la cualidad problematizadora que implica la investigación acción la coherencia entre la práctica, las teorías y aun los referentes institucionales de cada país pueden ser conciliados en la medida que el docente como ser autónomo se preocupe por un ejercicio contextualizado, Bernardo Restrepo (2003) en su artículo Aportes de la investigación - acción educativa a la hipótesis del maestro investigador asimila este accionar contextualizador con el término del filósofo francés Derida "Deconstrucción” la cual es considerada por su autor como

"la puesta en juego de los elementos de la estructura del texto para sacudirla, hallar sus opuestos, atacar el centro que la sostiene y le da consistencia, para hallarle las inconsistencias, volverla inestable y encontrarle un nuevo centro, que no será estable indefinidamente, pues el nuevo sistema puede contener inconsistencias que habrá que seguir buscando" (Derida citado en Restrepo 2003)

Evidentemente ese ir y venir en la identificación de inconsistencias es el ciclo en espiral que propone la reflexión acción que no se conforma con los resultados sin más si no que siempre está problematizando la realidad con el objetivo de mejorarla. Es así como en la sociedad de cambio constante del siglo XXI este método investigativo hace del acto educativo un accionar pertinente porque se preocupa por estar acorde con las necesidades educativas y exigencias de la sociedad actual y es en este contexto cambiante el profesorado tiene la ardua tarea de formar al ser humano lo que le confiere a su labor una gran complejidad si se tiene en cuenta la multidimensionalidad humana y "el surgimiento de demandas cambiantes y expectativas educacionales complejas en la sociedad de la información y el conocimiento" (Avalos, 2010).

Para finalizar el presente análisis cabe decir en palabras de Latorre (2001) que "la escuela del tercer milenio precisa de una enseñanza de calidad, pero no logrará tal objetivo si continua pasiva y libresca, erudita y poco crítica, ello significa que solo en el ejercicio investigativo en la acción se desarrollaran las herramientas necesarias para hacer de la educación un ejercicio pertinente frente a los cambios continuos dela sociedad del siglo XXI.

\section{CONCLUSIÓN}

Para concluir, no todas las metodologías de investigación sirven para indagar la práctica profesional; dependerá de los propósitos y de las metas que deseemos alcanzar. En el caso del profesorado, la finalidad es mejorar, innovar, comprender los contextos educativos teniendo como meta la calidad de la educación. (Latorre 2001) resumen: la IA es la metodología ideal para investigaciones de la práctica educativa cuyo objetivo este encaminado en mejorar la calidad de la educación 
El método de investigación acción en el área educativa:

Un ejercicio pertinente e inclusivo para los formadores del siglo XXI.

Monica Chicaiza Rey - Yesenia Martelo - Ronal Blanquicett Narváez (84:94)

Es así, que la investigación-acción es un método pertinente por que guía al investigador aparte de conocer una determinada realidad o un problema específico de un grupo, también desea darle solución. De esta manera, los sujetos a investigar participan como co-investigadores en todas las etapas del proceso: planteamiento del problema, recolección de la información, interpretación de indagación, planeación y cumplimiento de la acción concreta para dar solución al problema, evaluación posterior sobre lo realizado, entre otras. Por ende, la investigación-acción aplicada al área educativa es una manera de comprender la enseñanza, no sólo de investigar lo relacionado con ella.

De la misma manera, la investigación - acción y su aplicación en el área educativa supone entender la enseñanza como un proceso de investigación, un proceso de constante búsqueda. Lleva a una profunda reflexión sobre el oficio docente, integrando la concentración y el trabajo intelectual en las observaciones de las experiencias que se realizan, como un mecanismo esencial de la actividad educativa. Por ejemplo, los problemas orientan toda acción, pero lo primordial en la investigación-acción es la indagación reflexiva que el docente hace de su práctica, la planifique, visualice y tenga la capacidad de adaptar mejoras continuas. En general, la investigación - acción constituye una vía de reflexiones sistemática sobre la práctica con el fin de optimizar los procesos de enseñanza-aprendizaje.

En definitiva, para crear o aseverar en el campo de la cooperación, las técnicas de la investigación - acción han sido aplicadas y empleadas de manera asertiva por diferentes instituciones y entidades en campos del cooperativismo en diferentes niveles de la educación. En este sentido, es primordial resaltar las diferentes metodologías que conllevan a un creciente campo de los enfoques cooperativos y participativos que tiene como objetivo crear un proceso de sensibilización, concienciación y participación en torno a una problemática

En último término, la importancia de la cooperación en el siglo XXI residirá en la manera para transferir valores que faciliten la construcción de una sociedad justa, igualitaria, tolerante, pacifica, dinámica y diversa, asistiendo los recursos que proporciona el mismo contexto. De esta manera, sabemos que esto ya no es así. La educación requiere un vuelco rotundo de sus métodos y herramientas, pues la educación exige un mundo más dinámico, ágil y con múltiples posibilidades de proyección a la sociedad. Partiendo de un enfoque crítico, humanitario y transformador de la realidad se lleva a cabo una investigación-acción-participativa que con sus fases desarrollen diferentes procesos de acción-reflexión, en los que participen de forma conjunta investigadores, actores, en la caracterización de los problemas y en la obtención de propuestas y acciones de mejora. 
La cooperación en la investigación- acción comprende, por una parte, el inmenso potencial en la producción de conocimiento y en la relación de éste con la acción social, y por otro, el papel de la educación en el fortalecimiento de las estructuras educativas y comunitarias, conocimientos interdisciplinares y metodologías de trabajo que permiten obtener soluciones asertivas integrales y acordes a las necesidades educativas.

\section{REFERENCIAS BIBLIOGRÁFICAS}

Ávalos, B. (2007). El desarrollo profesional continuo de los docentes: lo que nos dice la experiencia internacional y de la región latinoamericana. Revista Pensamiento Educativo, 41; №2, 77-99. Retrieved from http://www.pensamientoeducativo.uc.cl/files/journals/2/articles/417/ public/417-934-1-PB.pdf

Balcázar, F. (2003). Investigación acción participativa (IAP): aspectos conceptuales y dificultades de implementación. Fundamentos En Humanidades, (7), 59-77.

E, A. M. C., \& M, M. L. P. (2008). LA INVESTIGACIÓN ACCIÓN. Una herramienta metodológica heurística para la comprensión y transformación de realidades y prácticas socioeducativas. Laurus, 14(27), 96-114.

Furlán, A., Ángel, M., Valdez, P., Nvestigación, I., Intervención, T. E., El, E. N., \& Pasillas, M. A. (1993). Perfiles Educativos.

John Elliott. (2005). La investigación-acción en educación. Perfiles Educativos, 1-20. Retrieved from https://www.worldcat.org/title/resena-de-la-investigacion-accion-en-educacionde-john-elliott/oclc/181381002\&referer=brief_results

Lier, L. (2001). La investigación-acción. Textos de Didáctica de La Lengua y La Literatura, pp. 81-88.

Perez, T. (n.d.). No Title. Pertinencia de La Educación: ¿pertinente Con Qué? Retrieved from https://www.mineducacion.gov.co/1621/article-209857.html

Rahman, A. y O. Fals (1992), "La situación actual y las perspectivas de la investigaciónacción participativa en el mundo", en Salazar, M. C., La investigación-acción participativa, ed. Popular, Madrid. 
El método de investigación acción en el área educativa:

Un ejercicio pertinente e inclusivo para los formadores del siglo XXI.

Monica Chicaiza Rey - Yesenia Martelo - Ronal Blanquicett Narváez (84:94)

Sirit, P., \& Enrique, A. (2014). Tendencias Epistemológicas de las Investigaciones en los Programas académicos De la división de Estudios para Graduados de la Facultad de ciencias Económicas y sociales De la universidad del Zulia. Orbis. Revista Científica Ciencias Humanas, 10(29), 6-36. 